BMJ Open Sport \& Exercise Medicine

\section{Sport and exercise medicine} consultants are reliable in assessing tendon neovascularity using ultrasound Doppler
To cite: Watson J,

Barker-Davies RM, Bennett AN, et al. Sport and exercise medicine consultants are reliable in assessing tendon neovascularity using ultrasound Doppler. BMJ Open Sport \& Exercise Medicine 2018;4:e000298. doi:10.1136/ bmjsem-2017-000298

JW and RMB-D are the jointfirst authors.

Accepted 13 December 2017

Check for updates

${ }^{1}$ Academic Department of Military Rehabilitation, Defence Medical Rehabilitation Centre, London, UK

${ }^{2}$ Cardiff School of Sport, Cardiff Metropolitan University, Cardiff, UK

${ }^{3}$ National Centre for Sport and Exercise Medicine, School of Sport Exercise and Health Sciences, Loughborough University, London, UK ${ }^{4}$ Faculty of Medicine, National Heart and Lung Institute, Imperial College, London, UK ${ }^{5}$ Athlete Health Department, English Institute of Sport, Manchester, UK

Correspondence to Dr Robert M Barker-Davies; robbarker-davies@doctors. net.uk

\section{ABSTRACT}

Objective Several lower limb tendinopathy treatment modalities involve identification of pathological paratendinous or intratendinous neovascularisation to target proposed co-location of painful neoneuralisation. The ability to reliably locate and assess the degree of neovascularity is therefore clinically important. The Modified Ohberg Score (MOS) is frequently used to determine degree of neovascularity, but reliability has yet to be established among Sport and Exercise Medicine (SEM) consultants. This study aims to determine inter-rater and intra-rater reliability of an SEM consultant cohort when assessing neovascularity using the 5-point MOS.

Method Eleven participants (7 male and 4 female) provided 16 symptomatic Achilles and patella tendons. These were sequentially examined using power Doppler (PD) enabled ultrasound (US) imaging by 6 SEM consultants who rated neovascular changes seen using the MOS. Representative digital scan images were saved for rescoring 3 weeks later. Inter-rater and intra-rater reliability of the MOS was examined using intraclass correlation coefficient (ICC) and Kappa Agreement scores. Results Neovascular changes were reported in $65.6 \%$ of 96 scans undertaken. ICC for inter-rater reliability was 0.86 and Fleiss Kappa 0.52. ICC for intra-rater reliability was 0.95 and Weighted Kappa 0.91.

Conclusions Neovascular changes were present in twothirds of symptomatic tendons. Excellent SEM consultant inter-rater and intra-rater reliability was demonstrated. These findings support the use of PD-enabled US to assess neovascularity by appropriately experienced SEM consultants. Furthermore, future interventional research using a similarly experienced SEM consultant cohort can be undertaken with assurance that assessment of neovascularity will be reliable.

\section{INTRODUCTION}

Incidence and prevalence of Achilles and patellar tendinopathies are increased in physically active populations. ${ }^{1-3}$ During the progression of tendinopathic disease, the formation and ingrowth of neovascular structures into the tendon is a widely recognised pathological hallmark. ${ }^{4-7}$ The role of neovascularisation within tendinopathy is the source

\section{What are the new findings?}

- Appropriately trained Sport and Exercise Medicine consultants have excellent inter-rater and intra-rater reliability using power Doppler enabled ultrasound to assess neovascularity in chronic Achilles and patellar tendinopathy.

- Previously, such results have only been demonstrated among sonographers and radiologists.

- Neovascularity is present in two-thirds of chronic Achilles and patellar tendinopathies.

of significant interest regarding its contribution to the pathophysiology of the injured and healing tendon. ${ }^{68}$ It may also be associated with neoneuralisation ${ }^{9}$ exacerbated by nociceptive stimuli such as glutamate ${ }^{10}$ and substance $\mathrm{P}^{11}$ This provides an explanation as to why the point of ingress of vessels into the tendon is often associated with the site of maximal pain on palpation. ${ }^{12}$ Neovascularisation is a normal physiological response in early repair, and its continued presence is likely to be a result of prolonged overload that delays the healing timeline. ${ }^{13}$ Association between neovascularity and pain and function has been shown in small interventional case and longitudinal studies ${ }^{14-17}$ yet refuted in Randomised Controlled Trial (RCT) ${ }^{18}$ and only weakly correlated in a larger prospective cohort. ${ }^{7}$ This supports the theory that neovascularity may be more a sign of chronicity than severity. Polidocanol ${ }^{14}$ and high volume image-guided injections (HVIGI) ${ }^{19}$ target the presence of vessels in order to address this proposed pain stimulus. ${ }^{14}{ }^{19}$ To that end, the ability to reliably locate and quantify vascularity is clinically relevant.

The hypoechoic areas seen in tendinopathy on gray-scale ultrasound (US) offer limited information which can be enhanced by the use of power Doppler (PD) to identify hyperaemic neovasculature. ${ }^{710142021} \mathrm{In}$ 
clinical practice, there have been 3 methods of assessing the degree of neovascularisation. The first is a simple statement of whether vessels are either absent or present in the tendon structure. ${ }^{8}$ Depending on the significance of degree of vascularity as discussed above, the utility of such binary grading is likely to be limited. A second system involves determining the PD signal surface area by pixel count. Although a seemingly robust method of capturing a quantifiable measure of neovascularity, these authors recognise that interpretation of findings in clinical practice will be both machine and operator setting dependent. ${ }^{223}$ A third method was pioneered by Ohberg and Alfredson ${ }^{14}$ who undertook PD US examination of symptomatic Achilles tendons to understand the extent of neovascularity and locate target areas for sclerosing polidocanol injections. In this study, an experienced sonographer assessed neovascularisation as $0,1+, 2+, 3+$ or $4+$. In the absence of vessels, the tendon scored a 0 . A score of $1+$ was ascribed if the tendon showed 1-2 small vessels primarily around the anterior surface of the tendon. Scores of $2+, 3+$ or $4+$ were given if the vessel contained 2, 3 or more than 4 vessels respectively throughout its structure. They later adapted this score for use in patellar tendinopathy with the score of $1+$ referring to $1-2$ vessels mostly in the posterior portion of that tendon. ${ }^{16}$

Sengkerij $e t a l^{21}$ undertook an inter-rater reliability study in which a cohort of radiologists undertook PD US examinations of symptomatic and asymptomatic Achilles tendons and rated the neovascularisation referring to the system described above as the Modified Ohberg Score (MOS). Of the 50 tendons examined in the study, each underwent scans by 2 of 8 radiologists. Excellent reliability was found with absolute agreement of $62 \%$ and an intraclass correlation coefficient (ICC) of 0.85 . Sunding $e t a l^{24}$ used a more qualitative 4-point MOS for neovascularity: 0 , None; 1 , Mild; 2, Moderate; 3 , Severe. They therefore used Kappa in their analysis of 27 Achilles and 26 patella tendons finding good inter-rater agreements of 0.63 and 0.70 respectively using 2 sonographers.

US scanning is a common skill used frequently in clinical practice, yet there are no data to support its reliability among Sport and Exercise Medicine (SEM) consultants. This study aims to contribute to future research by establishing reliability among SEM consultants when assessing tendon neovascularity using the 5-point MOS.

\section{METHOD}

Using a power of $80 \%(\beta$-level=0.8) and an $\alpha$-level of 0.05 anticipating good to excellent reliability $\left(\mathrm{P}_{0}>0.7\right.$ to $\left.\mathrm{P}_{1}=0.9\right)$, the calculations of Walter et $a l^{25}$ were used to estimate the requirement for at least 5 raters $(\mathrm{n})$ of 10 participants $(\mathrm{k})$.

\section{PARTICIPANTS}

All participants gave written informed consent. Participants self-identified following advertisement for volunteers with Achilles or patella tendon pain. A
Table 1 Inclusion and exclusion criteria

\begin{tabular}{ll}
\hline Inclusion criteria & Exclusion criteria \\
\hline 18-60years of age & Systemic \\
Clinical findings of Achilles or patellar & $\begin{array}{l}\text { condition causing } \\
\text { inflammatory }\end{array}$ \\
tendinopathy: & tendinopathy \\
Localised pain to tendon & \\
Pain/stiffness on waking & \\
Pain on commencing loaded activity & \\
Pain after activity & \\
Sain on mobilising after rest & \\
for period in excess of 2 months & \\
\hline
\end{tabular}

subjective and objective assessment from an experienced physiotherapist (JW) against inclusion/exclusion criteria (table 1) was conducted consecutively. Participants were requested to avoid any heavy sporting or loading activity 24 hours prior to US examination.

Six SEM consultant raters were recruited to the study having completed professional training for a mean of 8 years (range 1-23 years). Four had undergone additional postgraduate training in musculoskeletal US, while the remainder had attained significant clinical experience in scanning both Achilles and patella tendons.

\section{Data collection}

Prior to testing, the consultant raters received an instructional brief on the examination protocol which included standardised positioning of participants, surface pressure produced through the probe at the contact point, labelling of recorded scans and clarity as to the descriptions of the MOS (table 2). Participants were subjected to repeat PD US examination by all 6 raters.

Scans were performed using a Logic 9 US Scanner (GE Medical Systems, Chalfont St Giles, UK) with a high frequency linear ML 6-15 MHz transducer (GE Medical Systems). Consultants oriented themselves to the tendon using standard gray-scale US to identify areas of the tendon to focus subsequent PD scanning. Once PD mode was engaged, the first consultant when scanning a participant's tendon set and standardised the PD gain used for the subsequent consultants. The first consultant to scan was varied for each patient to control any bias in setting of PD gain. Where possible,

Table 2 Description of the 5-point Modified Ohberg Score

\begin{tabular}{ll}
$\begin{array}{l}\text { Modified Ohberg } \\
\text { Score }\end{array}$ & Description \\
\hline 0 & No vessels \\
$1+$ & $\begin{array}{l}\text { One vessel anterior to the Achilles } \\
\text { One vessel posterior to the patella tendon }\end{array}$ \\
$2+$ & One to two vessels throughout the tendon \\
$3+$ & $\begin{array}{l}\text { Three vessels throughout the tendon } \\
4+\end{array}$ \\
& $\begin{array}{l}\text { Four or more vessels throughout the } \\
\text { tendon }\end{array}$ \\
\hline
\end{tabular}




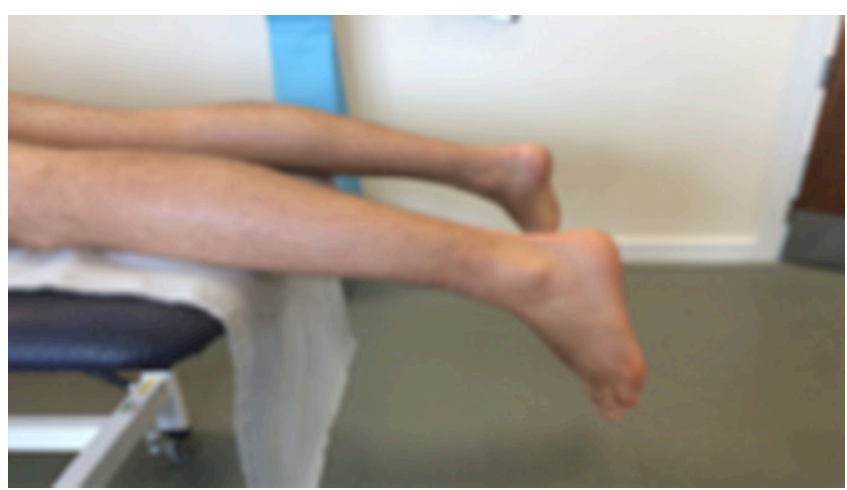

Figure 1 Standardised position for ultrasound imaging of the Achilles tendon.

participants were scanned in series by all 6 consultants within the same session. To control for changes in tendon loading that may affect vessel signal, participants were instructed to avoid heavy sporting or loading activity 24 hours prior to repeat examinations.

To prevent vessel compression, positioning of the tendon was strictly controlled. Achilles tendons were scanned in the prone position with the foot in a relaxed non-dorsiflexed position over the end of the treatment couch (figure 1). Patella tendons were scanned in a standardised position of approximately $30^{\circ}$ of knee flexion (figure 2A). Consultants were also permitted to scan in full knee extension (figure 2B) to remove any tension from the tendon.

\section{Data storage}

Participants were assigned an anonymised unique identifier used to save images on the Logic 9 Scanner. Scans were saved once each consultant determined the MOS. Consultants were requested to save any scans that contributed to them formulating an overall MOS for the tendon. All MOS scores were recorded on paper retained out of sight of other raters. Three weeks after the initial examination, raters rescored the saved scans. Clinical information including previous MOS ratings was not available to consultant raters at any stage.

\section{Statistical analysis}

Inter-rater and intra-rater reliability across the cohort of consultants was calculated using ICC and Kappa in IBM SPSS Statistics V.23 (IBM, Amonk, New York, USA) with a one-way random, single measures model. Fleiss defines
Table 3 Distribution of the Modified Ohberg Scores across all 96 examinations

\begin{tabular}{lll}
\hline $\begin{array}{l}\text { Modified Ohberg } \\
\text { Score }\end{array}$ & $\begin{array}{l}\text { Number of scores } \\
\text { recorded }\end{array}$ & $\begin{array}{l}\text { Percentage of } \\
\text { scores recorded }\end{array}$ \\
\hline 0 & 33 & 34.4 \\
\hline+ & 23 & 23.9 \\
$2+$ & 16 & 16.7 \\
$3+$ & 5 & 5.2 \\
$4+$ & 19 & 19.8 \\
\hline
\end{tabular}

an excellent ICC as higher than $0.75,{ }^{26}$ a good ICC as $0.4-0.75$ and poor if less than 0.4 . For clinical investigations, an ICC of 0.60 has been described as the minimally acceptable level. ${ }^{27}$ Fleiss Kappa statistic was used to measure the level of agreement between raters, while a weighted Kappa was used to allow marginal agreement between individual raters' baseline and follow-up scores. ${ }^{28}$ These were calculated using SPSS extension bundles. Both ICC and Kappa were used to provide comparability across the literature.

\section{RESULTS}

Eleven participants ( 7 males and 4 females with a mean age of 36.2 years (SD 6.25)) were recruited to the study and provided 16 symptomatic tendons. The mean duration of symptoms was 20.1 months (range 6-96 months).

\section{Presence of neovascularisation}

Eight symptomatic Achilles and 8 patella tendons $(n=16)$ were assessed by 6 consultant raters $(\mathrm{K}=6)$ resulting in 96 examinations. Neovascularisation was reported in $65.6 \%$ of scans and the MOS distribution is shown in table 3 .

\section{Inter-rater reliability}

In 6 tendons $(37.5 \%)$, there was absolute scoring agreement across all raters, and a further 6 tendons had agreement from at least 4 of the 6 raters. This represents an agreement of 4 or more consultant raters in $75 \%$ of the participants. The ICC for inter-rater reliability of the MOS was 0.86 (95\% CI, 0.76 to 0.94 ), illustrating excellent inter-rater reliability. The Fleiss Kappa agreement for inter-rater reliability was 0.52 (95\% CI, 0.45 to 0.59 ). It should be noted the more modest result
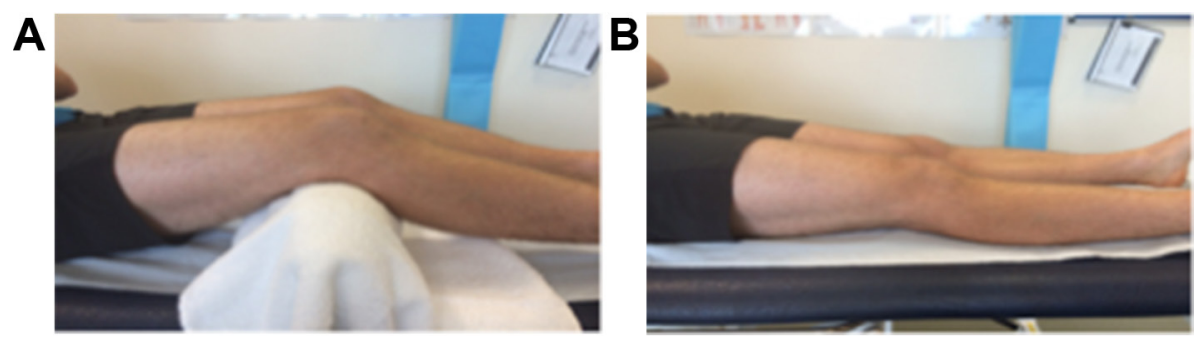

Figure 2 (A)Standardised position for US imaging of the patella tendon (position 1). (B) Standardised position for US imaging of the patella tendon (position 2). US, ultrasound. 
Table 4 Agreement between baseline and follow-up scoring

\begin{tabular}{lllllll}
\hline \multicolumn{7}{c}{ Follow-up scan } \\
& & 0 & $1+$ & $2+$ & $3+$ & $4+$ \\
& 0 & 29 & 3 & 0 & 0 & 0 \\
Baseline & $1+$ & 1 & 19 & 2 & 0 & 1 \\
scan & $2+$ & 0 & 2 & 14 & 0 & 1 \\
& $3+$ & 0 & 0 & 0 & 4 & 1 \\
& $4+$ & 0 & 0 & 0 & 0 & 18 \\
\hline
\end{tabular}

of Fleiss Kappa is not weighted and based on absolute agreement.

\section{Intra-rater reliability}

Comparison of the agreement between baseline and follow-up scores can be found in table 4. Across raters absolute agreement between initial and follow-up scans occurred in 84 of 95 scans (one scan did not save), representing $88 \%$ of scans undertaken.

The ICC for intra-rater reliability within the cohort was 0.95 (95\% CI, 0.92 to 0.97$)$ illustrating excellent reliability of the MOS when reviewing scans. The weighted Kappa agreement score was 0.91 (95\% CI 0.85 to 0.97) demonstrating excellent reliability.

\section{DISCUSSION}

This study has demonstrated excellent inter-rater reliability (ICC 0.86) of a cohort of SEM consultants when using the MOS to rate neovascularisation in Achilles and patella tendons. Furthermore, excellent intra-rater reliability (ICC 0.95) was demonstrated via review of saved scans. Neovascularisation was reported in $65.6 \%$ of the original scans in keeping with previous studies of Achilles and patellar tendinopathy. ${ }^{21}$ 29-31 These results suggest that diagnostic US scoring of tendinopathic neovascularisation can be reliably employed in the SEM setting. The most important variable that will allow results to be extrapolated to a wider audience is the degree of clinical exposure to tendon assessment and ultrasonography clinicians have undertaken. All clinicians involved in this study described themselves as highly competent in the ultrasonography of Achilles and patella tendons, although this was not corroborated independently the results are comparable with those reported using radiologist or sonographer raters. ${ }^{21} 24$

In terms of distribution of MOS, it can be noted (table 3) that there was a lower number of Grade $3+$ scores attributed to any of the primary scans undertaken. This is in keeping with the findings of Sengkerij and colleagues ${ }^{21}$ who found that only $9 \%$ of their scans received a $3+$ score. An explanation for this is possibly that the raters find it easy to identify $1-2$ vessels within the tendon structure, but once vascularity is more prevalent, it may be difficult to differentiate the existence of additional single vessels, the result being a lean towards a higher score of $4+$. As the results of this study agree with those of Sengkerij et $a l^{21}$ it may provide evidence to suggest that the 5-point scale is reduced to a 4-point scale, with the final score (grade 3) representing 3 or more vessels.

This study builds on the work of Sengkerij $e t a l^{21}$ who did not attempt to control any of the operator variables once the PD setting was enabled. Of particular importance in this respect is the gain setting, which regulates the sensitivity of the system to flow. ${ }^{32}$ Essentially, too high a gain and the amount of random noise artefact in the form of colour foci in the image will increase, potentially affecting the clarity of colour that indicates vascular flow. In this study, the first consultant to scan a given participant was asked to select and set what they considered to be an appropriate gain, which other consultants would then use and this stayed consistent for that participant. As a result, the sensitivity of the system was standardised for each participant. Sengkerij et $a l^{21}$ used 2 radiologists each scanning session of a larger cohort $(n=8)$. In such an uncrossed design, ICC does not readily deal with missing data. Using default SPSS settings with a one-way random ICC model cases that are not scanned by all raters would be excluded from the analysis. One solution to this would be to arrange data in 2 columns giving the appearance of a full data set from 2 raters exclusively. Collapsing data across empty columns and unequal proportions of scans undertaken by raters could overestimate the inter-rater ICG they reported of 0.85 , though such limitations can only be assumed.

Arguably, the MOS is only a semiquantitative scale; therefore, this study has also used Fleiss and weighted Kappa. These favourable results compare well with Sunding et al who reported Kappas of 0.63 and 0.70 in Achilles and patellar tendinopathy, respectively using 2 independent sonographers. ${ }^{24}$ Weighted Kappa, which, like ICC, recognises marginal agreement and does not extend to comparison of more than 2 data sets. This presents a problem for Kappa analysis of more than 2 raters. The commonly used Fleiss Kappa result in this study should be interpreted differently from the other results because it can only reward absolute agreement. Light's Kappa, which is the mean of all rating pairs, ${ }^{33}$ is a different solution yet not often reported. Therefore, the Fleiss Kappa has been presented in this study alongside an excellent inter-rater ICC, which has been able to provide context.

A strength of this study was the ability to recruit an appropriately powered number of consultant raters and where possible have them available for sequential scanning of all participants. Shoukri et $a t^{27}$ noted the difficulty in recruiting a number of suitably qualified specialists within the Hospital setting. While a large cohort of SEM consultants undertook ratings, short notice changes in availability were a limitation. The initial design aim for each participant to be scanned by 6 consultants sequentially in one session was achieved for 9 of 16 tendons. The remainder had to undertake subsequent scanning sessions (within a mean of 7.8 days). Boesen et $a t^{22}$ reported an increase 
in vessel appearance as an acute response to exercise, although postulate that this may be short-lived. As a result, those participants that were required to return for subsequent scans were requested to refrain from any activity that would either aggravate their symptoms or potentially make an acute change to the vessel appearance. This variable would have a potential to reduce consultant agreement on their primary scans of each patient, but despite this, excellent reliability was still demonstrated.

\section{FURTHER RESEARCH}

The findings of this study have direct implications for future research and clinical practice. Primarily, a subcohort of the consultants in this study are also involved in a large $\mathrm{RCT}^{34}$ to investigate the effects of HVIGI (with and without steroid) on chronic Achilles and patella tendon pain. The neovessels found on scanning of the participants will be targeted by the injections delivered, and therefore this reliability study serves to provide assurance that the consultants are reliable when scoring neovasculature presence and extent. Furthermore, it can be extrapolated that consultants of similar experience to those in this study will provide reliable PD US assessments for future studies using the MOS, as either an outcome measure or target for intervention. In the clinical setting, this study provides evidence to support the use of diagnostic US in SEM clinical practice by proving reliability both in initial scanning and in the test-retest situation, such as follow-up scanning postintervention.

\section{CONCLUSION}

This study demonstrated excellent inter-rater and intrarater reliability of the scoring of neovasculature within tendon structure among SEM consultants. While this contributes to the clinical assessment of patients with tendon pain, it does not explain the role of neovasculature in the pathological process or as a source of pain in chronic tendinopathy. Well-controlled research is required to establish whether procedures targeting neovascularisation can effect improvements in validated pain and functional outcome measures.

Contributors JW and RMB-D contributed equally in study design, ethical approval, study implementation, clinical protocols, drafting and editing of the manuscript. ANB is responsible for study design, clinical protocols, ethical approval, clinical and scientific supervision of manuscript drafting, editing and review. DTPF, ML and $\mathrm{CR}$ are responsible for study design, scientific protocols, clinical and scientific supervision, manuscript editing and review. PCW is responsible for study design, clinical protocols, scientific protocols, clinical and scientific supervision, manuscript editing and review. All authors read and approved the final manuscript.

Funding This study has attracted funding through Loughborough University from the Higher Education Funding Council for England and a PhD Studentship.

\section{Competing interests None declared.}

Ethics approval Ethical approval was obtained via both the Ministry of Defence Research and Ethics Committee (684/MODREC/15) and the Cardiff Metropolitan University School of Sport Research Ethics Committee.

Provenance and peer review Not commissioned; externally peer reviewed.

Open Access This is an Open Access article distributed in accordance with the terms of the Creative Commons Attribution (CC BY 4.0) license, which permits others to distribute, remix, adapt and build upon this work, for commercial use, provided the original work is properly cited. See: http://creativecommons.org/ licenses/by/4.0/

(c) Article author(s) (or their employer(s) unless otherwise stated in the text of the article) 2018. All rights reserved. No commercial use is permitted unless otherwise expressly granted.

\section{REFERENCES}

1. de Jonge S, van den Berg C, de Vos RJ, et al. Incidence of midportion Achilles tendinopathy in the general population. $\mathrm{Br} J$ Sports Med 2011;45:1026-8.

2. Zwerver J, Bredeweg SW, van den Akker-Scheek I. Prevalence of Jumper's knee among nonelite athletes from different sports: a cross-sectional survey. Am J Sports Med 2011;39:1984-8.

3. Van Middelkoop M, Kolkman J, Van Ochten J, et al. Prevalence and incidence of lower extremity injuries in male marathon runners. Scand J Med Sci Sports 2008;18:140-4.

4. Cook JL, Purdam CR. Is tendon pathology a continuum? A pathology model to explain the clinical presentation of load-induced tendinopathy. Br J Sports Med 2009;43:409-16.

5. Malliaras P, Purdam CR. Proximal hamstring tendinopathy assessment and management. Sports Health 2014;32:21-9.

6. Rees JD, Wilson AM, Wolman RL. Current concepts in the management of tendon disorders. Rheumatology 2006;45:508-21.

7. De Jonge S, Warnaars JL, De Vos RJ, et al. Relationship between neovascularization and clinical severity in Achilles tendinopathy in 556 paired measurements. Scand J Med Sci Sports 2014;24:773-8.

8. Ohberg L, Lorentzon R, Alfredson H. Neovascularisation in Achilles tendons with painful tendinosis but not in normal tendons: an ultrasonographic investigation. Knee Surg Sports Traumatol Arthrosc 2001;9:233-8.

9. Webborn AD. Novel approaches to tendinopathy. Disabil Rehabil 2008;30:1572-7.

10. Alfredson $\mathrm{H}$. Chronic midportion Achilles tendinopathy: an update on research and treatment. Clin Sports Med 2003;22:727-41.

11. Lian Ø, Dahl J, Ackermann PW, et al. Pronociceptive and antinociceptive neuromediators in patellar tendinopathy. Am J Sports Med 2006;34:1801-8.

12. Divani K, Chan O, Padhiar N, et al. Site of maximum neovascularisation correlates with the site of pain in recalcitrant midtendon Achilles tendinopathy. Man Ther 2010;15:463-8.

13. Ackermann PW. Neuronal regulation of tendon homoeostasis. Int $J$ Exp Pathol 2013;94:271-86.

14. Ohberg L, Alfredson $H$. Ultrasound guided sclerosis of neovessels in painful chronic Achilles tendinosis: pilot study of a new treatment. $\mathrm{Br}$ $J$ Sports Med 2002;36:173-5.

15. Ohberg L, Alfredson H. Sclerosing therapy in chronic Achilles tendon insertional pain-results of a pilot study. Knee Surg Sports Traumatol Arthrosc 2003;11:339-43.

16. Alfredson $\mathrm{H}$, Ohberg L. Neovascularisation in chronic painful patellar tendinosis-promising results after sclerosing neovessels outside the tendon challenge the need for surgery. Knee Surg Sports Traumatol Arthrosc 2005;13:74-80.

17. Cook JL, Malliaras P, De Luca J, et al. Vascularity and pain in the patellar tendon of adult jumping athletes: a 5 month longitudinal study. Br J Sports Med 2005;39:458-61.

18. Hoksrud A, Ohberg L, Alfredson H, et al. Color Doppler ultrasound findings in patellar tendinopathy (jumper's knee). Am J Sports Med 2008;36:1813-20.

19. Chan O, O'Dowd D, Padhiar N, et al. High volume image guided injections in chronic Achilles tendinopathy. Disabil Rehabil 2008;30:1697-708.

20. Newman JS, Adler RS, Bude RO, et al. Detection of soft-tissue hyperemia: value of power Doppler sonography. AJR Am J Roentgenol 1994;163:385-9.

21. Sengkerij PM, de Vos RJ, Weir A, et al. Interobserver reliability of neovascularization score using power Doppler ultrasonography in midportion achilles tendinopathy. Am J Sports Med 2009;37:1627-31.

22. Boesen MI, Koenig MJ, Torp-Pedersen S, et al. Tendinopathy and Doppler activity: the vascular response of the Achilles tendon to exercise. Scand J Med Sci Sports 2006;16:463-9.

23. Malliaras $P$, Chan $O$, Simran G, et al. Doppler ultrasound signal in Achilles tendinopathy reduces immediately after activity. Int J Sports Med 2012;33:480-4.

24. Sunding K, Fahlström M, Werner S, et al. Evaluation of Achilles and patellar tendinopathy with greyscale ultrasound and colour Doppler: 
using a four-grade scale. Knee Surg Sports Traumatol Arthrosc 2016;24:1988-96.

25. Walter SD, Eliasziw M, Donner A. Sample size and optimal designs for reliability studies. Stat Med 1998;17:101-10.

26. Fleiss J. The design and analysis of clinical experiments. New York: Wiley, 1986.

27. Shoukri MM, Asyali MH, Donner A. Sample size requirements for the design of reliability study: review and new results. Stat Methods Med Res 2004;13:251-71.

28. Robinson G, O'Donoghue P. A weighted kappa statistic for reliability testing in performance analysis of sport. Int $J$ Perform Anal Sport 2007;7:12-19.

29. Zanetti M, Metzdorf A, Kundert HP, et al. Achilles tendons: clinical relevance of neovascularization diagnosed with power Doppler US. Radiology 2003;227:556-60.
30. Peers KH, Brys PP, Lysens RJ. Correlation between power Doppler ultrasonography and clinical severity in Achilles tendinopathy. Int Orthop 2003;27:180-3.

31. Reiter M, Ulreich N, Dirisamer A, et al. Colour and power Doppler sonography in symptomatic Achilles tendon disease. Int J Sports Med 2004;25:301-5.

32. Torp-Pedersen ST, Terslev L. Settings and artefacts relevant in colour/power Doppler ultrasound in rheumatology. Ann Rheum Dis 2008;67:143-9.

33. Hallgren KA. Computing Inter-Rater Reliability for Observational Data: An Overview and Tutorial. Tutor Quant Methods Psychol 2012;8:23-34.

34. Barker-Davies RM, Nicol A, McCurdie I, et al. Study protocol: a double blind randomised control trial of high volume image guided injections in Achilles and patellar tendinopathy in a young active population. BMC Musculoskelet Disord 2017;18:204 